\title{
Estructura del fitoplancton en las épocas seca y lluviosa en el golfo de Papagayo, Costa Rica
}

\section{Phytoplankton structure in dry and rainy seasons in the gulf of Papagayo, Costa Rica}

\author{
Sandra Loza Álvarez ${ }^{1}$, Rosario Benavides-Morera ${ }^{2}$, Carlos Luis Brenes-Rodríguez ${ }^{2 *}$ y \\ Daniel Ballestero Saxon ${ }^{2}$
}

\section{RESUMEN}

Con el propósito de estudiar la estructura de la comunidad fitoplanctónica en el golfo de Papagayo, se realizaron 2 muestreos en febrero y junio del 2017, y se analizó adicionalmente información hidrográfica. Su composición estuvo dominada, en ambas épocas, por diatomeas, dinoflagelados, cianobacterias y flagelados. La fracción de diatomeas aportó mayoritariamente a la estructura del fitoplancton con 41 taxa, luego los dinoflagelados con 20. En las diatomeas, los géneros más abundantes y con mayor frecuencia de aparición fueron Rhizosolenia sp., Guinardia sp. y Thalassiosira sp. En los dinoflagelados, Tripos furca predominó en la estación 20 en Bahía Culebra y resultó frecuente Gyrodiniumspirale, así como los géneros Gymnodinium sp., Gyrodinium sp. y Amphidinium sp. Las cianobacterias estuvieron representadas, principalmente, por formas chroocoides de los géneros Anacystis sp. y Chroococcus sp., al igual que las haptoficeas y los flagelados, por los coccolitoforidos y el género Chrysocromulina sp., respectivamente. En febrero, la concentración fitoplanctónica varió entre 326 y 1063 cél mL $\mathrm{mL}^{-1}$, y en junio entre 653 y 1041 cél $\mathrm{mL}^{-1}$, con características mesotróficas en ambas épocas. La hidrografía estuvo regulada por el ciclo anual de precipitaciones y el afloramiento estacional costero de Papagayo. En febrero, respecto a octubre, la temperatura superficial descendió $3^{\circ} \mathrm{C}$, la salinidad ascendió 2 y la concentración de clorofila- $a$ aumentó en $0.5 \mathrm{mg} \cdot \mathrm{m}^{-3}$. Aguas más cálidas, menos salinas y con menores concentraciones de clorofila- $a$ en octubre $\left(\mathrm{T}>29^{\circ} \mathrm{C}, \mathrm{S}<32.2\right.$, Chl- $\left.a<0.5 \mathrm{mg} \mathrm{m}^{-3}\right)$ contrastan con lo observado en febrero.

Palabras claves: Zona costera, diatomeas, dinoflagelados, cianobacterias, hidrografía.

\section{ABSTRACT}

In order to study the phytoplankton structure in the gulf of Papagayo, 2 sampling cruises were conducted in February and June of 2017, and hydrographic information was additionally analyzed. The phytoplankton composition was dominated in both months by diatoms, dinoflagellates, cyanobacteria and flagellates. The fraction of diatoms contributed mainly to the phytoplankton structure

1 Instituto de Ciencias del Mar de Cuba.

2 Laboratorio de Oceanografía y Manejo Costero, Universidad Nacional, Heredia, Costa Rica, Apdo. 86-3000. sloza@ceniai.inf.cu, rosario.benavides.morera@una.ac.cr, carlos.brenes.rodriguez@una.ac.cr*,dab2@una.ac.cr 
with 41 taxa, followed by dinoflagellates with 20 taxa. In diatoms, the genera with the greatest contribution to diversity and frequency were Rhizosolenia sp., Guinardia sp., and Thalassiosira $\mathrm{sp}$. In the case of dinoflagellates, Tripos furca predominated in station 20 located in Culebra Bay, while Gyrodiniumspirale was frequent, as well as genera Gymnodinium sp., Gyrodinium sp., and Amphidinium sp. Cyanobacteria were mainly composed by genera Analysis sp. and Chroococcus sp. and haptoficeas and flagellates, by the Coccolitoforids and the genus Chrysocromulina sp., respectively. In February the phytoplankton concentration varied between 326 and 1063 cells $\mathrm{mL}^{-1}$, and in June between 653 and 1041 cells $\mathrm{mL}^{-1}$, with mesotrophic characteristics in both seasons. The gulf's hydrography is regulated by the annual rainfall cycle and the seasonal coastal upwelling of Papagayo. In February, as opposed to October, surface temperature decreased by $3^{\circ} \mathrm{C}$, salinity increased by 2 and chlorophyll- $a$ concentration increased by $0.5 \mathrm{mg} . \mathrm{m}^{-3}$. Warmer waters, with lower saline and chlorophyll-a concentrations in October $\left(\mathrm{T}>29^{\circ}, \mathrm{S}<32.2\right.$, Chl-a $<0.5 \mathrm{mg} \mathrm{m}^{-3}$ ) are opposed to February results.

Keywords: Coastal zone, diatoms, dinoflagellates, cyanobacteria, hydrography.

\section{INTRODUCCIÓN}

Con una extensión de $180 \mathrm{~km}^{2}$, el golfo de Papagayo (GP), localizado en el Pacífico Norte de Costa Rica, se ubica desde Punta Gorda hasta la península de Santa Elena. Una importante extensión de este se integra al Sistema de Parques Nacionales. Su profundidad varía hasta los $100 \mathrm{~m}$ y su climatología muestra una estacionalidad bien definida entre las épocas seca (diciembre-marzo) y lluviosa (mayo-noviembre) (IMN, 2008).

Al norte del GP, la periodicidad de las surgencias costeras, causadas por el forzamiento de los vientos predominantes del noroeste en la superficie del mar, influyen de manera estacional sobre su hidrografía (Ballestero et al. 2012) y, probablemente, sobre las concentraciones de fitoplancton en dicho golfo, con la consecuente transmisión de energía en niveles tróficos superiores.
El GP comprende varios ecosistemas costeros muy interesantes $\mathrm{y}$, al mismo tiempo, poco estudiados. Solo existe un trabajo que hace énfasis en la comunidad fitoplanctónica (Morales et al. 2001), a través del abordaje de un evento de marea roja en Bahía Culebra y Playa Panamá, ambos dentro del GP. En los últimos años, los usos de la zona costera del GP se han diversificado e intensificado, convirtiéndolo en una de las áreas más prósperas de la costa noroccidental del país, cuya mayor potencialidad se expresa en el turismo y la pesca. Adicional al trabajo antes citado, en el área solo existen estudios ambientales (May-Collado \& Morales, 2005; Salas, 2010; Vargas, 2013) y se adolece de una caracterización integral de sus aguas desde el punto de vista oceanográfico-biológico, que permita, entre otras cosas y mediante un enfoque ecológico, realizar en un futuro cercano la evaluación de la calidad de sus aguas. 
En este sentido, la comunidad fitoplanctónica es utilizada para evaluar la salud de los ecosistemas (De la Lanza et al. 2000; Loza et al. 2007; Jozwiak et al. 2008; Simon et al. 2009). El fitoplancton se ha destacado como un indicador de la calidad del agua cuando es analizado en el nivel de población y comunidad. Ha resultado útil en la identificación de especies clave para la comunidad y regiones de importancia biológica, proveyendo, de esta manera, un fundamento para el monitoreo de los posibles cambios de un determinado ecosistema, asociados al régimen climático e hidrológico, a diferentes escalas temporales y espaciales (Herrera-Silveira et al. 1999).

De este modo, el presente trabajo tiene como objetivo, en primera instancia, la caracterización de la comunidad fitoplanctónica en el golfo de Papagayo, en los períodos seco y lluvioso, y su relación con la hidrografía de la zona. Se busca, adicionalmente, contribuir con el inicio de una línea base de conocimiento, que a futuro permita monitorear y evaluar los cambios que eventualmente sucedan en este ecosistema marino, producto de la actividad humana o por fenómenos asociados a la variabilidad climática.

\section{METODOLOGÍA}

Los muestreos en el golfo de Papagayo se llevaron a cabo en octubre del año 2016 y tanto febrero como junio del año 2017, meses representativos de las épocas seca y lluviosa, y en las cuales existen marcadas diferencias en la dinámica y la climatología de la zona. Los levantamientos hidrográficos se realizaron con un perfilador de temperatura, salinidad y densidad (CTD) modelo SB-19 plus, el que, adicionalmente, tenía sensores para la determinación de clorofila y oxígeno disuelto. Se muestreó en 24 estaciones, de las cuales se seleccionaron 8 para el estudio del fitoplancton, procurando, en este último caso, abarcar 3 zonas bien diferenciadas: la región con influencia más oceánica $(2,9$ y 18$)$, la más costera $(5,13$ y 14) y la posible área con mayor impacto antropogénico como lo es Bahía Culebra (20 y 24) (Fig. 1).

Los datos de precipitación entre enero del 2016 y mayo del 2017 provienen de un pluviómetro electrónico Campbell, cuyas lecturas son horarias y pertenecen a la estación localizada en Nacascolo, en el centro-sur del golfo de Papagayo, en las coordenadas $10^{\circ} 38^{\prime} \mathrm{N}, 85^{\circ} 39^{\prime} \mathrm{W}$, a una elevación de $1 \mathrm{msnm}$.

Las muestras de agua para los análisis de fitoplancton se colectaron a $25 \mathrm{~m}$ de profundidad en el máximo de clorofila- $a$, excepto en las estaciones 5 y 20 , donde la profundidad local era inferior a $30 \mathrm{~m}$.

Para determinar la composición de la comunidad fitoplanctónica, las muestras fueron colectadas en botellas Niskin de 1 litro, utilizando lugol ácido 


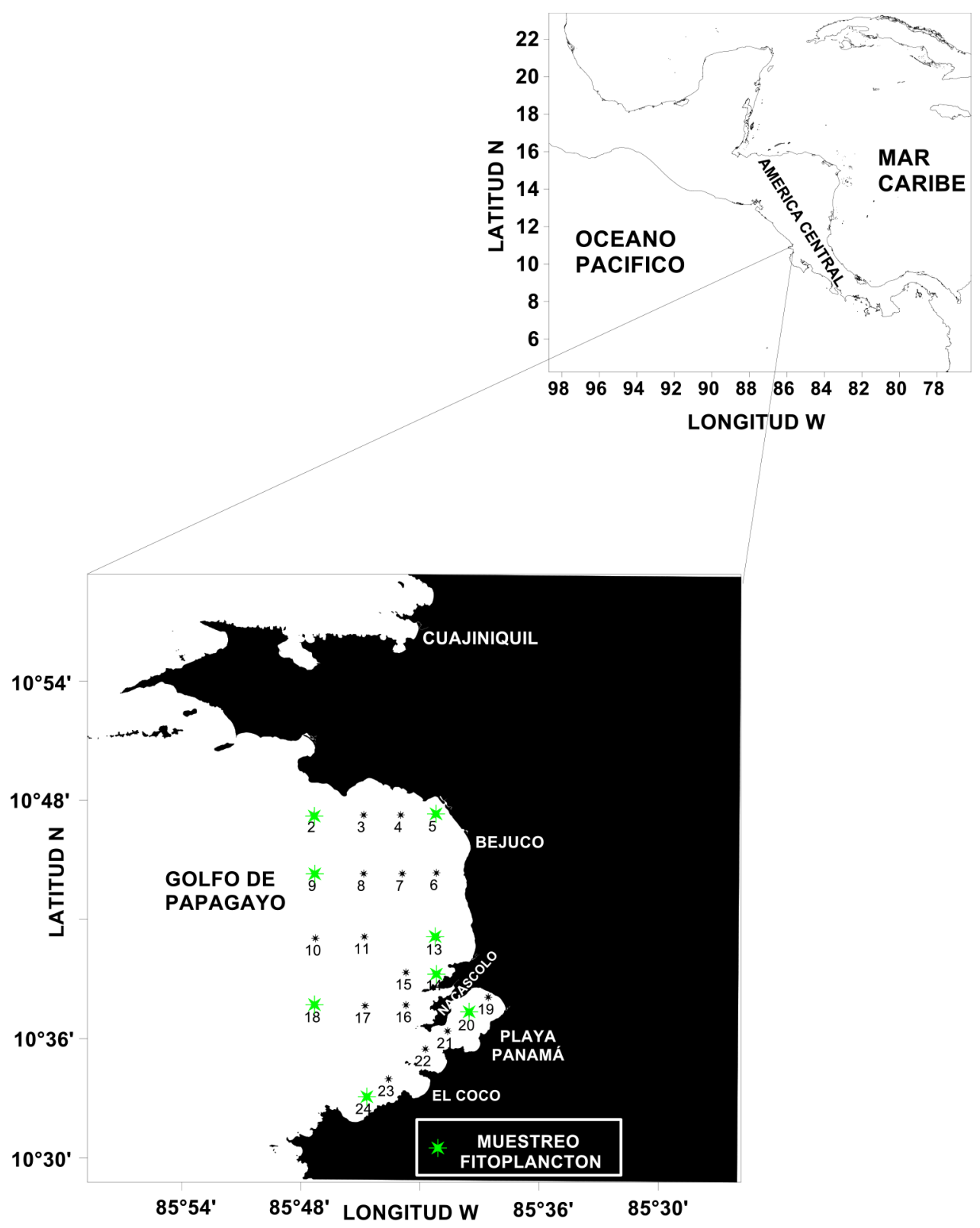

Fig. 1. Ubicación de las estaciones de muestreo hidrográfico y de fitoplancton Fig. 1 Location of hydrographic and phytoplankton sampling station 
como fijador. En el laboratorio fueron procesadas según la metodología propuesta por Miravet et al. (2009). El análisis al microscopio se realizó a partir de una submuestra de $0.02 \mathrm{~mL}$, con ayuda de un microscopio Olympus y un aumento de $400 \mathrm{x}$. Para el inventario de los organismos, se observó todo el volumen de la submuestra y, para su cuantificación, se escogieron líneas al azar en una cámara Sedgwick-Rafter.

Con el propósito de establecer las diferentes categorías taxonómicas, se adoptó el sistema de clasificación según el sitio algaebase.org (Guiry \& Guiry, 2017). La identificación de los taxones se realizó de acuerdo con Licea-Durán et al. (1995), Moreno-Ruíz et al. (1996), Tomas (1997), Sant'Anna et al. (2005) y Gómez (2013).

Se utilizó la concentración de fitoplancton para definir las características tróficas de las aguas, utilizando la escala de Kloblents-Mishke \&Vedernikov (1979).

Para determinar las diferencias entre estaciones y épocas de acuerdo con la concentración fitoplanctónica, se empleó la escala propuesta por Frontier (1981), la cual se basa en el análisis estadístico de las denominadas clases de abundancia establecidas sobre una progresión geométrica de razón 4.3. Las distribuciones espaciales y los perfiles verticales de las variables fí- sicas fueron realizados utilizando SURFER 14 (2017) y GRAPHER 8 (2009).

\section{RESULTADOS}

La Figura 2 muestra los valores mensuales de precipitación en la estación de Nacascolo. En junio y octubre ocurren los 2 máximos anuales de lluvia en esta región ( 275 y 240 mm, respectivamente), así como, entre enero-abril, el período más seco, con un promedio mensual del orden de los $12 \mathrm{~mm}$.

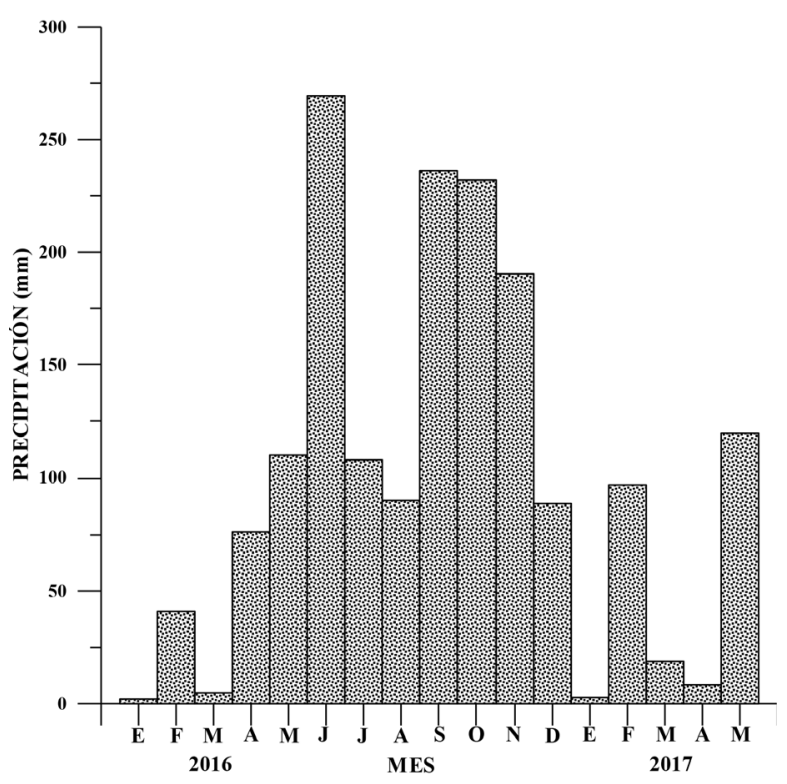

Fig. 2. Precipitación mensual (mm) en la estación de Nacascolo entre enero del 2016 y mayo del 2017

Fig. 2. Monthly precipitation (mm) at the Nacascolo Station between January 2016 and May 2017 
La Figura 3 presenta las distribuciones superficiales de temperatura, salinidad y clorofila- $a$ para octubre del 2016 y febrero del 2017. En época seca (febrero), las 3 variables muestran valores superficiales diferentes a los obtenidos en época lluviosa (octubre). En febrero, la temperatura (Fig. 3B) varió desde un mínimo de $25.3^{\circ} \mathrm{C}$ hasta un valor máximo de $27.5^{\circ} \mathrm{C}$, de modo que el campo térmico en este estrato estuvo hasta $3^{\circ} \mathrm{C}$ por debajo de lo observado en octubre (Fig. 3A). En el caso de la salinidad, esta variable mostró su máximo valor en febrero (33.9) (Fig. 3D), con valores por encima de lo observado en octubre (Fig. 3C), al igual que la concentración de clorofila- $a$ con $0.5 \mathrm{mg}$ . $\mathrm{m}^{-3}$ (Fig. 3F) más que en octubre (Fig. $3 \mathrm{E})$. Aguas más cálidas, menos salinas y con menores concentraciones de clorofila- $a$ en octubre $\left(\mathrm{T}>29^{\circ} \mathrm{C}, \mathrm{S}<32.2\right.$, Chl- $a<0.5 \mathrm{mg} \mathrm{m}^{-3}$ ) contrastan con lo observado en febrero.

En época lluviosa, la zona central y más externa del golfo muestra temperaturas inferiores a $29.1^{\circ} \mathrm{C}$ (Fig. $3 \mathrm{~A}$ ) y salinidades menores a 31.5 (Fig. 3C). En esta misma zona, se observa un máximo de clorofila- $a$ cercano a 1 $\mathrm{mg} \mathrm{m}^{-3}$, al igual que en Bahía Culebra (Fig. 3F). En febrero, las temperaturas superficiales son menores en los extremos norte y sur del golfo (Fig. 3B) $\left(\mathrm{T}<27^{\circ} \mathrm{C}\right)$. Nuevamente, la parte central presenta las temperaturas más elevadas. En el caso particular de la salinidad, las aguas frías de la región norte están asociadas a salinidades altas $(\mathrm{S}>33.5)$, y en Bahía Culebra se tienen los valores más bajos $(\mathrm{S}<32$ ) (Fig. 3D). El campo superficial de clorofila- $a$ se muestra bastante homogéneo en febrero, con valores del orden de $1.1 \mathrm{mg}$ $\mathrm{m}^{-3}$ en casi todo el golfo, excepto, nuevamente, en el extremo norte donde esta variable supera los $1.4 \mathrm{mg} \mathrm{m}^{-3}$ (Fig. 3F). En las figuras $4 \mathrm{~A}$ y $4 \mathrm{~B}$, se observan los perfiles verticales de la temperatura, densidad y clorofila- $a$ en la estación 24 (Fig. 1), correspondientes a octubre del 2016 y junio del 2017, respectivamente. Se presenta, entre épocas, una diferenciada distribución vertical en la clorofila- $a$, en la cual el máximo ubicado entre los 25 y $35 \mathrm{~m}$ de profundidad en octubre desaparece en febrero, para dar lugar a un estrato homogéneo entre la superficie y los 25 $\mathrm{m}$ con concentraciones hasta 3 veces mayores que las de octubre.

La comunidad fitoplanctónica estuvo mayoritariamente representada por 4 grupos: diatomeas, dinoflagelados, cianobacterias y flagelados. En cuanto al número de especies, se observó un predominio de diatomeas y dinoflagelados (cuadros 1 y 2). La fracción de las diatomeas fue la que más aportó a la riqueza específica del fitoplancton con 41 taxa, seguida de los dinoflagelados con 20 taxa (Cuadro 1). En el grupo de los flagelados, se logró identificar únicamente el género Chrysocromulina sp. 

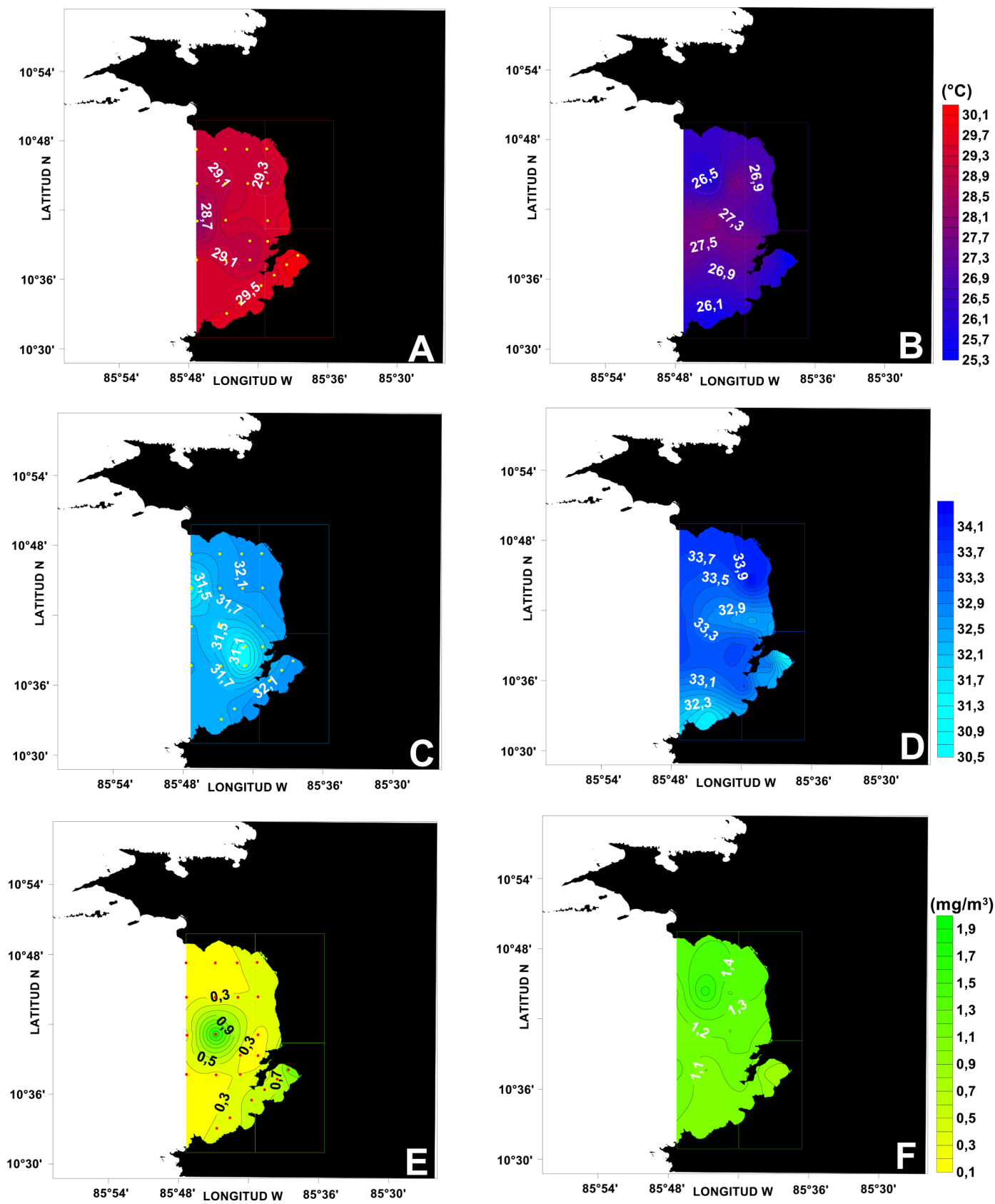

Fig. 3. Distribuciones superficiales de temperatura $\left({ }^{\circ} \mathrm{C}\right)(\mathrm{A}, \mathrm{B})$, salinidad $(\mathrm{C}, \mathrm{D}) \mathrm{y}$ clorofila-a (mg m-3) (E, F) para los meses de octubre del 2016 y febrero del 2017 Fig. 3. Surface distributions of temperature $\left({ }^{\circ} \mathrm{C}\right)(\mathrm{A}, \mathrm{B})$, salinity $(\mathrm{C}, \mathrm{D})$ and chlorophyll-a (mg m-3) (E, F) for October 2016 and February 2017 

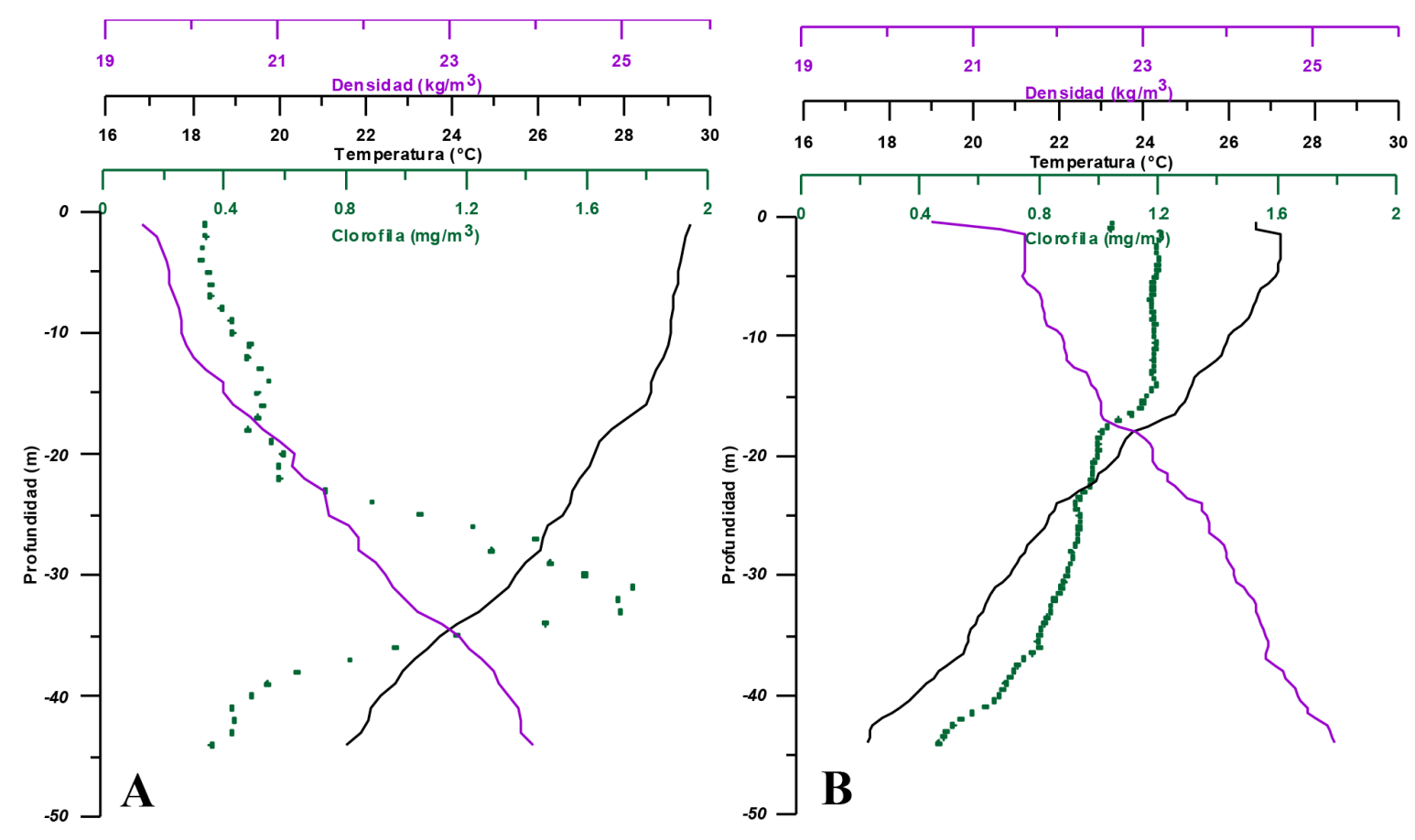

Fig. 4. Perfiles verticales de temperatura, densidad y clorofila-a en la estación 24, correspondientes a los meses de octubre del 2016 (A) y febrero del 2017 (B) Fig. 4. Vertical profiles of temperature, density and chlorophyll-a at station 24 for the months of October 2016 (A) and February 2017 (B)

En época lluviosa (junio), la riqueza específica de las diatomeas presentó un aumento en todas las estaciones, la de los dinoflagelados resultó muy similar en las 2 épocas (Cuadro 2). En las diatomeas, los géneros con mayor frecuencia de aparición fueron Rhizosolenia sp., Guinardia sp., Thalassiosira sp. y Guinardia sp. Entre los dinoflagelados, la especie Tripos furca predominó en la estación 20, ubicada en Bahía Culebra, y resultaron frecuentes la especie Gyrodiniumspirale y los géneros Gymnodinium sp., Gyrodinium sp. y Amphidinium sp. (Cuadro 1).

Las cianobacterias estuvieron representadas, principalmente, por 2 géneros: Anacystis sp. y Chroococcus sp., cuya frecuencia de aparición no varió entre estaciones ni entre épocas, y las haptoficeas y los flagelados estuvieron representados por los coccolitoforidos y el género Chrysocromulina sp., respectivamente (Cuadro 1). 
$\cdot \frac{\pi}{0}$
0
0
0
0
0
0
0
0
0
0
0

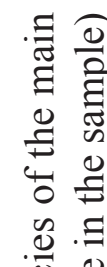

클유 융 है

$\cdot \frac{0}{0}$

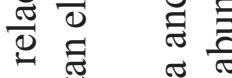

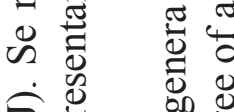

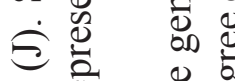

을 Ð

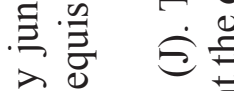

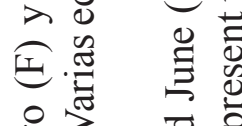

율 छ छ

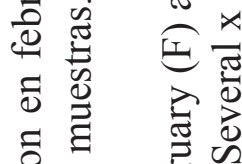

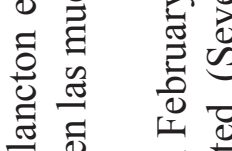

总.

ర

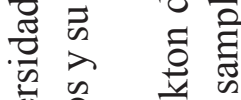

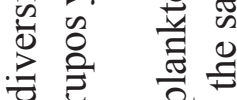

- 50 용.

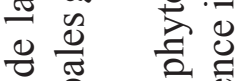

을 फे क्षै

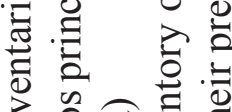

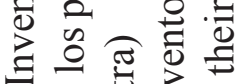

$\therefore$ 元芯

임 $\frac{d}{0}$ 光 -

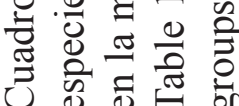
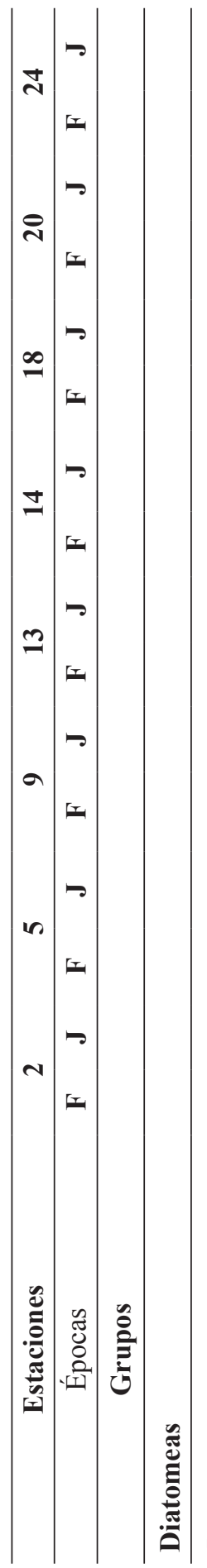

$x$

REVISTA CIENCIAS MARINAS Y COSTERAS 


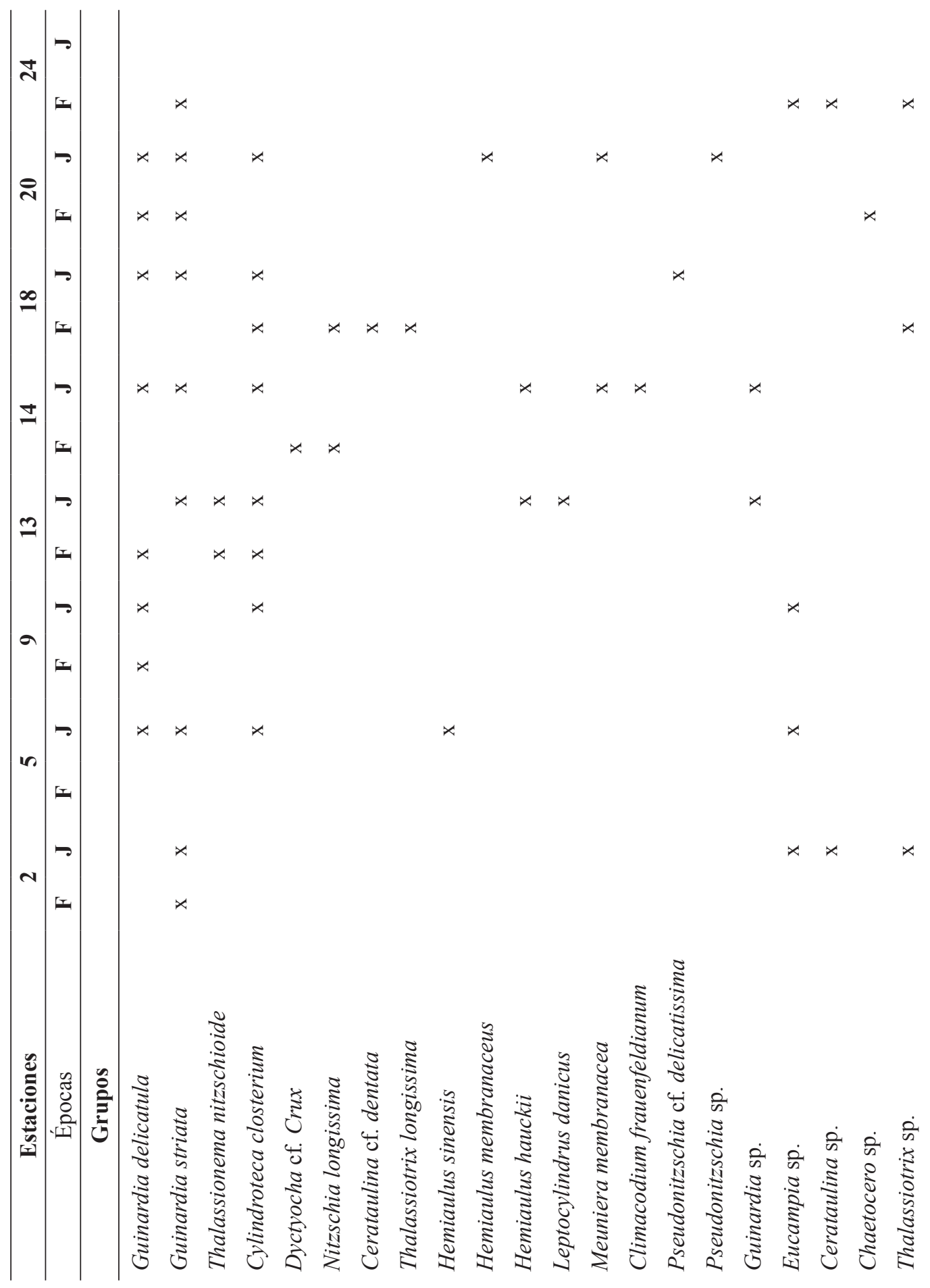


Estructura del fitoplancton en las épocas seca y lluviosa en el golfo de Papagayo,

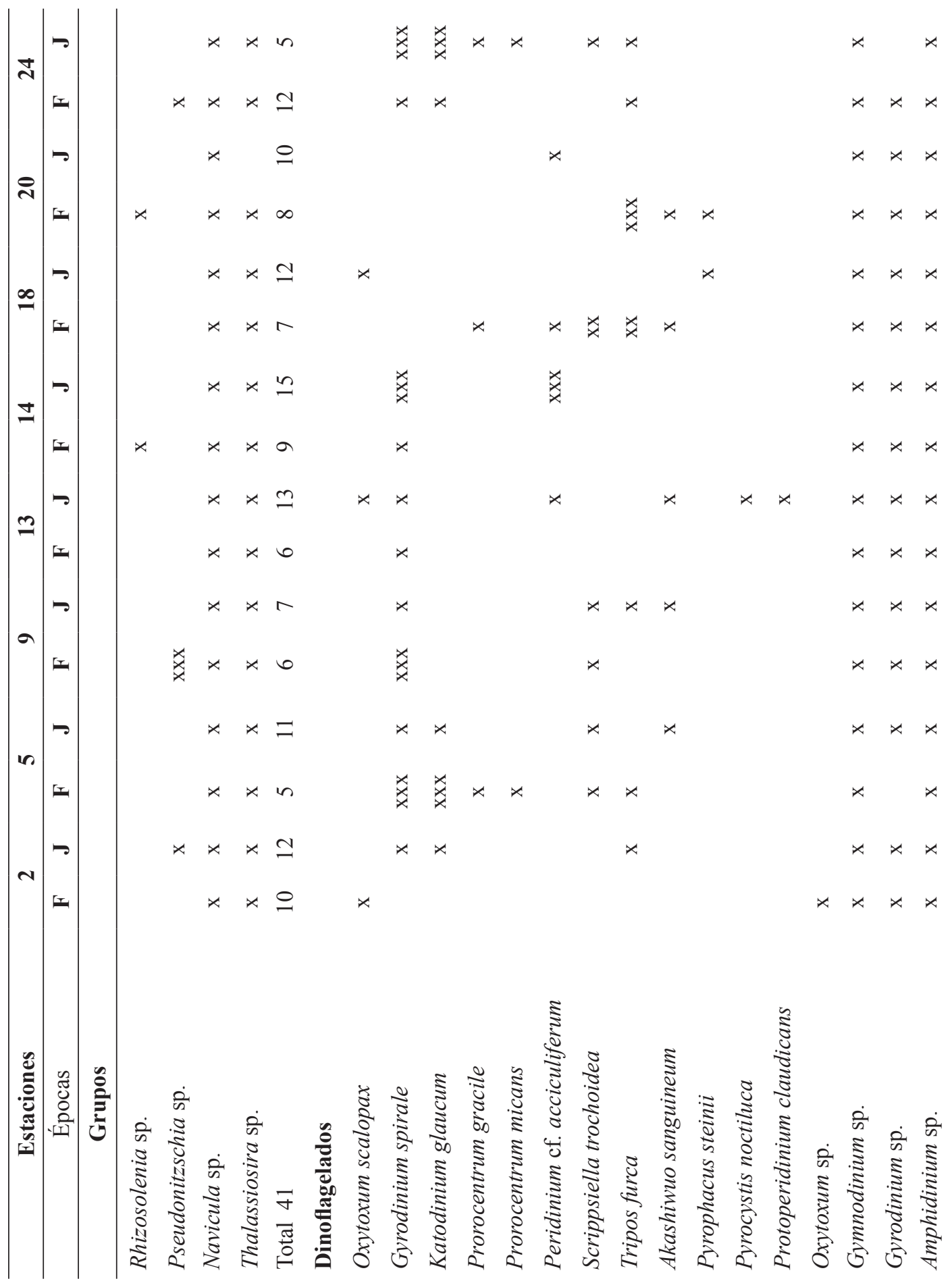




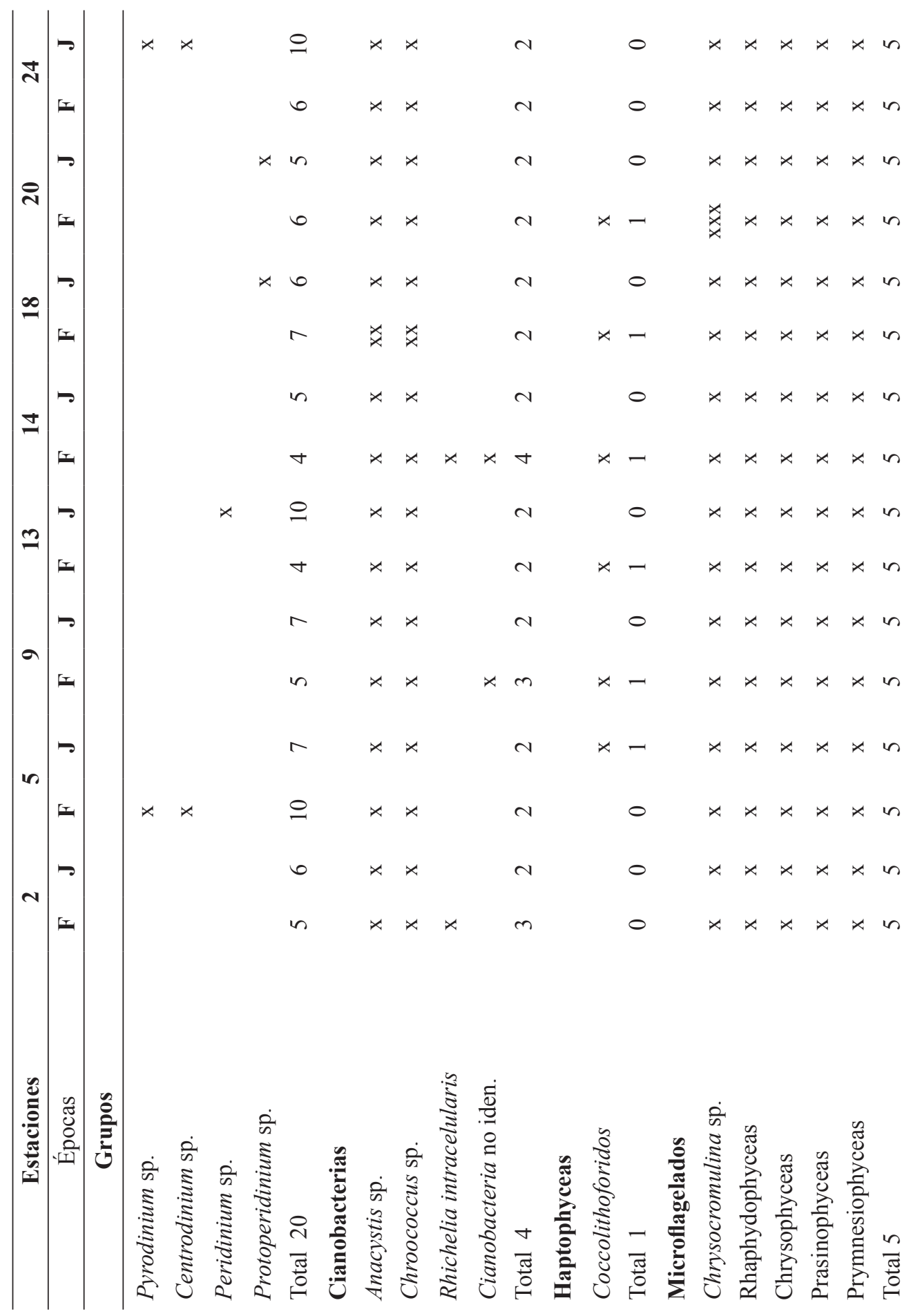


Cuadro 2. Géneros y especies del fitoplancton identificados en época seca (febrero) y época lluviosa (junio) en el golfo de Papagayo

Table 2. Phytoplankton genera and species identified during the dry season (February) and rainy season (June) in the gulf of Papagayo

\begin{tabular}{|c|c|c|c|c|c|c|}
\hline & Grupos & $\begin{array}{c}\text { Diato- } \\
\text { meas }\end{array}$ & $\begin{array}{c}\text { Dinoflagela- } \\
\text { dos }\end{array}$ & $\begin{array}{c}\text { Cianobacte- } \\
\text { rias }\end{array}$ & $\begin{array}{c}\text { Haptofi- } \\
\text { cea }\end{array}$ & Flagelados \\
\hline Estaciones & Época & & & & & \\
\hline \multirow{2}{*}{2} & Seca & 10 & 5 & 3 & 0 & 1 género \\
\hline & Lluviosa & 12 & 6 & 2 & 0 & 1 género \\
\hline \multirow{2}{*}{5} & Seca & 5 & 10 & 2 & 0 & 1 género \\
\hline & Lluviosa & 11 & 7 & 2 & 1 & 1 género \\
\hline \multirow{2}{*}{9} & Seca & 6 & 5 & 3 & 1 & 1 género \\
\hline & Lluviosa & 7 & 7 & 2 & 0 & 1 género \\
\hline 13 & Seca & 6 & 4 & 2 & 1 & 1 género \\
\hline 14 & Lluviosa & 13 & 10 & 2 & 0 & 1 género \\
\hline \multirow{2}{*}{18} & Seca & 9 & 4 & 4 & 1 & 1 género \\
\hline & Lluviosa & 15 & 5 & 2 & 0 & 1 género \\
\hline \multirow{2}{*}{20} & Seca & 7 & 7 & 2 & 1 & 1 género \\
\hline & Lluviosa & 12 & 6 & 2 & 0 & 1 género \\
\hline \multirow{2}{*}{24} & Seca & 8 & 6 & 2 & 1 & 1 género \\
\hline & Lluviosa & 10 & 5 & 2 & 0 & 1 género \\
\hline
\end{tabular}

En febrero la concentración fitoplanctónica varió entre 326 y 1063 cél $\mathrm{mL}^{-1}\left(\mathrm{X}_{\text {media }}=563 \pm 157\right.$ cél $\mathrm{mL}^{-1}$ desviación estándar), y en junio entre 653 y 1041 cél $\mathrm{mL}^{-1}\left(\mathrm{X}_{\text {media }}=848 \pm 144\right.$ cél $\mathrm{mL}^{-1}$ desviación estándar) (Fig. 5). Hubo un incremento de la abundancia de fitoplancton en junio, de hasta 2 órdenes de magnitud, con respecto a febrero (Fig. 5), exceptuando la estación 20 , donde se encontró en febrero el máximo valor para los 2 muestreos
(1063 cél $\mathrm{mL}^{-1}$ ), superando lo encontrado en cualquier otra estación. La aplicación de la escala de Frontier (1981) permitió determinar que solo los valores de abundancia en la estación 2 fueron significativamente menores en ambos meses, respecto al resto de las estaciones. La concentración promedio total del fitoplancton fue un $43 \%$ mayor en junio en comparación con febrero (Fig. 5). 
En febrero, las cianobacterias presentaron los mayores valores de concentración en las estaciones 18, 20 y 24, ubicadas hacia la parte sur del golfo y en Bahía Culebra, mientras que en período lluvioso se encontraron en las estaciones 2 y 5 , localizadas en la parte norte del golfo de Papagayo. En esta misma época, la estación 20 dentro de Bahía Culebra se distingue del resto por presentar las mayores concentraciones en los 4 grupos (Fig. 1, Cuadro 3). El máximo valor de dinoflagelados observado en esta estación en febrero estuvo determinado por una gran cantidad de organismos de la especie Tripos furca (242 cél $\left.\mathrm{mL}^{-1}\right)$, los cuales representaron el $75 \%$ de la población cuantificada de dinoflagelados (Cuadro 1).
La contribución en términos de porcentaje de los grupos a la concentración total del fitoplancton estuvo dominada por el aporte de los flagelados en ambas épocas (32\%-58\%) (Cuadro 4). Las cianobacterias aumentaron su aporte de manera general en época seca (febrero), las diatomeas mostraron un incremento en su contribución en época lluviosa (junio) y, finalmente, los dinoflagelados aumentaron su aporte en época seca, en las estaciones más al norte ( 2,5 y 9$)$, y en las estaciones más al sur, incluyendo Bahía Culebra, el comportamiento fue inverso $(14,18,20$ y 24) (Cuadro 4).

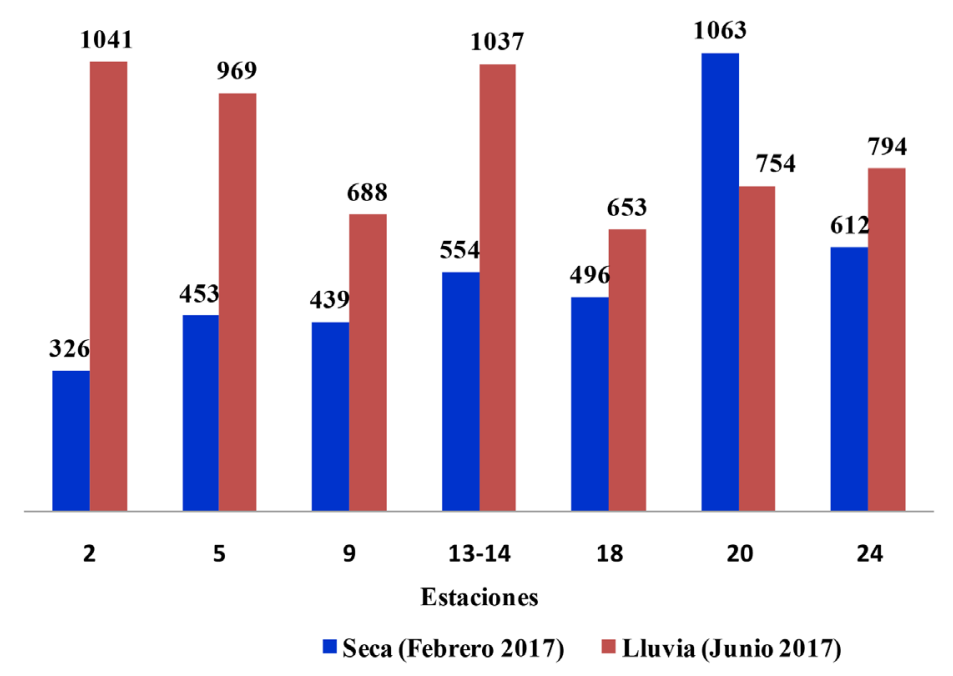

Fig. 5. Concentración de fitoplancton (cél $\mathrm{mL}-1$ ) en época seca (febrero) y época lluviosa (junio) en el golfo de Papagayo

Fig. 5. Concentration of phytoplankton (cel mL-1) during the dry season (February) and rainy season (June) in the gulf of Papagayo 
Cuadro 3. Concentración de los principales grupos del fitoplancton en febrero y junio. Valores máximos, mínimos, promedio $\mathrm{y} \pm$ desviación estándar

Table 3. Concentration of major phytoplankton groups in February and June. Maximum, minimum, mean values and \pm standard deviation

\begin{tabular}{ccccccccc}
\hline Estaciones & \multicolumn{2}{c}{ Diatomeas } & \multicolumn{2}{c}{ Dinoflagelados } & \multicolumn{2}{c}{ Cianobacterias } & \multicolumn{2}{c}{ Flagelados } \\
\hline & Febrero & Junio & Febrero & Junio & Febrero & Junio & Febrero & Junio \\
\hline 2 & 22 & 71 & 31 & 107 & 131 & 271 & 142 & 592 \\
5 & 22 & 103 & 127 & 105 & 150 & 267 & 154 & 492 \\
9 & 34 & 36 & 90 & 124 & 65 & 126 & 250 & 402 \\
13 & 21 & 109 & 76 & 265 & 129 & 90 & 322 & 573 \\
18 & 15 & 147 & 48 & 124 & 186 & 176 & 246 & 206 \\
20 & 61 & 101 & 324 & 101 & 233 & 120 & 445 & 432 \\
24 & 28 & 84 & 50 & 124 & 205 & 193 & 325 & 393 \\
Promedio/DE & $29 \pm 14$ & $93 \pm 32$ & $107 \pm 94$ & $136 \pm 54$ & $157 \pm 52$ & $178 \pm 66$ & $269 \pm 98$ & $441 \pm 121$ \\
Máx. & 61 & 147 & 324 & 265 & 233 & 271 & 445 & 592 \\
Mín. & 15 & 36 & 31 & 101 & 65 & 90 & 142 & 206 \\
\hline
\end{tabular}

Cuadro 4. Porcentaje (\%) de contribución de los principales grupos a la concentración total del fitoplancton en febrero y junio

Table 4. Contribution percentage ( $\%)$ of the main groups to the total concentration of phytoplankton in February and June

Grupos Diatomeas Dinoflagelados Cianobacte- Flagelados rias

\begin{tabular}{cccccc}
\hline Estaciones & Época & & & & \\
\hline \multirow{2}{*}{2} & Seca & 7 & 10 & 40 & 44 \\
& Lluviosa & 7 & 10 & 26 & 57 \\
\multirow{2}{*}{5} & Seca & 5 & 28 & 33 & 34 \\
& Lluviosa & 11 & 11 & 28 & 51 \\
\multirow{2}{*}{9} & Seca & 8 & 21 & 15 & 57 \\
\multirow{2}{*}{13} & Lluviosa & 5 & 18 & 18 & 58 \\
14 & Seca & 4 & 14 & 23 & 58 \\
\multirow{2}{*}{18} & Lluviosa & 11 & 26 & 9 & 55 \\
& Seca & 3 & 10 & 38 & 50 \\
20 & Lluviosa & 23 & 19 & 27 & 32 \\
& Seca & 6 & 30 & 22 & 42 \\
\multirow{2}{*}{24} & Lluviosa & 13 & 13 & 16 & 57 \\
& Seca & 5 & 8 & 33 & 53 \\
\hline
\end{tabular}


Al aplicar la escala de Kloblents-Mishke \& Vedernikov (1979) a las concentraciones de fitoplancton encontradas, se pudo concluir que las aguas del golfo de Papagayo presentan características mesotróficas en la mayoría de las estaciones. Solo las estaciones 5 , en febrero, y la 2 y la 14 , en junio, presentaron características eutróficas (Fig. 5).

\section{DISCUSIÓN}

Las distribuciones espaciales de temperatura, salinidad y clorofila- $a$ para octubre (lluvioso) (Fig. 3A, C, E) y febrero (seco) (Fig. 3B, D, F) son un reflejo de los factores que regulan la hidrografía del golfo de Papagayo: el ciclo anual de precipitaciones y el afloramiento estacional costero de $\mathrm{Pa}$ pagayo asociado al patrón de vientos.

Las condiciones climáticas en esta parte del Pacífico Norte de Costa Rica están dominadas por la migración norte-sur de la Zona de Convergencia Intertropical (ZCIT). Según el Instituto Meteorológico Nacional (IMN, 2008), el período seco en el Pacífico Norte se extiende de diciembre a marzo, meses en los cuales llueve únicamente el $4 \%$ de la precipitación anual, y el viento que predomina es el alisio del noreste; el mes de transición es abril y las lluvias inician en mayo. El 50\% de la precipitación anual ocurre entre mayo $\mathrm{y}$ agosto, y se observa en junio un máximo de lluvia. Un segundo pico de precipitación sucede entre setiembre y noviembre, meses en los cuales se presenta el restante $46 \%$ de la lluvia anual. Nuestros resultados (Fig. 2) coinciden con el análisis del comportamiento climatológico de la precipitación descrita por el Instituto Meteorológico Nacional (IMN, 2008).

Entre noviembre y marzo, el Pacífico Norte de Costa Rica y, de una manera muy directa, el GP se ven influenciados por el afloramiento costero de Papagayo que ocurre justo al norte de la zona estudiada (Chelton et al. 2000a; 2000b; Ballestero \& Coen, 2004; Ballestero et al. 2012; Fiedler \& Talley, 2006).

En superficie, los gradientes horizontales de las 3 variables ( $\mathrm{T}, \mathrm{S}$ y Cla) son en general más intensos en la dirección norte-sur que aquellos perpendiculares a la costa (Fig. 3). Esta característica es más evidente en época seca, cuando el extremo norte del golfo está frecuentemente influenciado por el afloramiento antes citado, lo cual permite zonificar latitudinalmente el golfo.

En este contexto, las bajas salinidades superficiales de octubre (lluvioso) con valores inferiores a 32.2 se asocian al aumento en las precipitaciones sobre el golfo, de la misma manera que las altas salinidades en febrero ( $\mathrm{S}$ $>33.1$ ) con la ausencia casi total de lluvias en la zona. Adicionalmente, en febrero, la disminución de la temperatura superficial y el aumento en las 
concentraciones de clorofila- $a$ y salinidades en dicho estrato (Fig. 3B, D, E) se explican también por la presencia de aguas de carácter oceánico en el golfo, cuyos índices termohalinos se ven modificados en esta época, debido al afloramiento de Papagayo (Brenes et al. 2008).

El análisis de los perfiles verticales de temperatura, densidad y clorofila- $a$ de la Figura 4B refuerza la tesis anterior. Dichos perfiles revelan, en febrero, condiciones oceánicas en el golfo con temperaturas superficiales inferiores a $27^{\circ} \mathrm{C}$. La isoterma de $25^{\circ} \mathrm{C}$ que identifica el tope de la termoclina en la región se localiza a una profundidad de $15 \mathrm{~m}$, unos $20 \mathrm{~m}$ por encima de su profundidad típica en el Pacífico Tropical Oriental (Fiedler, 2002), tal y como se puede observar en octubre $(35 \mathrm{~m})$ (Fig. 4A). Este hecho evidencia la introducción de agua subsuperficial en las capas superiores del mar. Adicionalmente, el máximo de clorofila- $a$, ubicado a una profundidad de $35 \mathrm{~m}$ en octubre (Fig. 4A), desaparece en febrero (Fig. 4B), para dar paso a una distribución verticalmente homogénea en los estratos más superficiales de la columna con valores más altos, poniendo de manifiesto, nuevamente, la intromisión de aguas subsuperficiales desde los niveles intermedios hacia la superficie (por encima de los $15 \mathrm{~m}$ de profundidad). Esta homogeneidad de los niveles más superficia- les en febrero refleja cómo la ausencia de fuentes laterales de agua dulce, la mezcla turbulenta inducida por el viento y los procesos de afloramiento son los factores moduladores de la estructura vertical de la columna de agua durante el período seco.

Como se mencionó en el párrafo precedente, el gradiente vertical de clorofila- $a$ está mucho más marcado en octubre que en febrero, con la presencia de un máximo a profundidades intermedias (Fig. 4A). Estos máximos subsuperficiales están asociados a una mayor disponibilidad de nutrientes en dichos niveles y a un aumento de la penetración de la luz, lo cual ha sido relacionado con la disminución de la concentración de plancton en superficie (Estrada, 1982). En febrero, con una columna de agua más mezclada y más densa (menor temperatura y mayor salinidad), se registra un aumento en la concentración superficial de clorofila- $a$ (Fig. 4B).

La comunidad fitoplanctónica (Cuadro 3) presentó una composición típica de zonas costeras, con un elevado aporte de especies de diatomeas y dinoflagelados, así como con elevadas concentraciones de flagelados (Moreira et al. 2007). Este comportamiento se relaciona con el mayor enriquecimiento en nutrientes y materia orgánica, propio de estas zonas (Caron, 2000). Las diatomeas se distinguen por presentar mayores requerimientos de nutrientes y los flagela- 
dos poseen la capacidad de degradar la materia orgánica (Potapova, 1993). No obstante, las elevadas concentraciones de fitoplancton de la estación 20, en febrero (Fig. 5), podrían responder a una posible contaminación (orgánica o inorgánica) existente en Bahía Culebra, producto de la antropización de la zona (Wo Ching \& Moreno, 2001; Sánchez-Noguera, 2012). El enriquecimiento de las aguas por escorrentía favorece la presencia de diatomeas en época lluviosa (junio), ya que este grupo no solo presenta mayores requerimientos de nutrientes esenciales, sino también de silicatos (Tomas, 1997).

En la composición de la comunidad fitoplanctónica encontrada en este estudio, se identificaron géneros y especies reportados como potencialmente nocivos y cianobacterias en todas las estaciones analizadas, lo cual se podría asociar, eventualmente, a una contaminación inorgánica, ya que estos organismos constituyen indicadores adecuados de alteraciones ambientales. Los organismos reportados fueron Scripsiella acuminata, Akashiwo sanguinea y Prorocentrum sp., los cuales se consideran potencialmente nocivos (Sar et al. 2002). También se observaron organismos indicadores de posible contaminación orgánica (saprobiedad), como es el caso de la especie de diatomea Nitzschialongissima y del género Chroococcus sp., los cuales están asociados a condiciones de contaminación moderada o betamesosaprobiedad, con valores de abundancia relativa de hasta un $50 \%$ en algunas estaciones.

En aguas del mar Mediterráneo, Friligos \& Koussouris (1984), al igual que Friligos (1989), asociaron a los dinaflagelados Gymnodinium sp., Prorocentrummicans, Peridinium sp. y Ceratium sp. con la presencia de contaminación producto de vertederos de aguas residuales. Los géneros Gonyaulax y Gymnodinium han sido reportados con un número importante de especies formadoras de mareas rojas tóxicas (Smayda \& Shimizu, 1993).

Vilicic et al. (1995), en un estudio realizado en la Bahía Gruz en el mar Adriático, observaron que las variaciones en las condiciones hidrográficas, asociadas principalmente a cambios en los patrones de escorrentía, tenían una influencia directa con los cambios observados en las comunidades de diatomeas, específicamente de Chaetoceros sp., Leptocylindrus sp., Nitzschiaseriata y Skeletonemacostatum. Las variaciones en el régimen de precipitación que caracteriza nuestra zona de estudio (Fig. 2), con diferencias en los acumulados de precipitación superiores a los $200 \mathrm{~mm}$ entre los meses lluviosos y secos, modularían el comportamiento de la hidrografía de la zona estudiada, con la respectiva influencia sobre la composición del fitoplancton entre épocas.

La composición del fitoplancton obtenida en ambas épocas pone de manifiesto su utilidad como un indicador 
sensible para evaluar la calidad de las aguas, a partir de los cambios que ocurren en el medio marino. El predominio de la especie Tripos furca encontrada en la estación 20 (Bahía Culebra), en época seca, podría tomarse como un resultado que justifique el monitoreo periódico del fitoplancton en esa área, ante la posibilidad de ocurrencia de florecimientos fitoplanctónicos, tal y como han sido reportados en el pasado (Morales et al. 2001). Estos autores reportaron la presencia del dinoflagelado Lingulodinium polyedrum por primera vez en Bahía Culebra, dentro del golfo de Papagayo y en Playa Panamá, el cual ha sido asociado con la producción de yesotoxinas, las cuales producen síntomas parecidos a los de las toxinas paralizantes (Paz et al. 2008).

Como se discutió en párrafos precedentes, el golfo de Papagayo experimenta estacionalmente la influencia del afloramiento costero que ocurre en esta región. Estos fenómenos son capaces de aumentar los niveles de clorofila (Fig. 3F) en el área y también los procesos turbulentos en la columna de agua, los cuales inducen, en algunos casos, la remoción de sedimentos en el lecho marino. Tal condición había sido reportada con anterioridad por Morales et al. (2001), al apuntar que los hipnoquistes de $L$. polyedrum necesitan un tiempo prolongado de maduración, al ser susceptibles de ser enterrados en los sedimentos antes de germinar, de modo que estas surgencias periódicas se convierten en mecanismos de resuspensión no solo de los nutrientes, sino de los hipnoquistes, manteniéndolos en la zona fótica durante el tiempo necesario para que germinen.

Por lo anterior, el objetivo de este trabajo no es evaluar el estado de salud ambiental del GP, sino hacer una descripción de la composición del fitoplancton presente en sus aguas, como un aporte a esta área de conocimiento, ya de por sí escaso para esta región. Sin embargo, a pesar de que la presencia de organismos potencialmente nocivos en nuestros análisis no es concluyente en cuanto al deterioro de dicho cuerpo de agua, sí puede contribuir a la creación de una línea base de resultados que permita, en el futuro cercano, planificar trabajos de investigación en esta zona costera de tanto interés en el campo de la preservación ambiental.

\section{AGRADECIMIENTOS}

Esta investigación se llevó a cabo con fondos de la Universidad Nacional de Costa Rica, a través de los proyectos Servicio Regional de Información Oceanográfica y Cambios estacionales de la calidad del agua del golfo de Papagayo, Guanacaste, Costa Rica. Nuestro agradecimiento a MarViva, por financiar los costos de la embarcación; a la Asociación Costa Rica por Siempre; al personal del Área de Conservación Guanacaste, y al Centro de Recursos Hídricos para 
Centroamérica y el Caribe (HIDROCEC), por el apoyo logístico para realizar el trabajo de campo.

\section{BIBLIOGRAFÍA}

Ballestero, D. \& Coen, E. (2004). Generation and propagation of anticyclonic rings in the Gulf of Papagayo, Costa Rica. Int. J. Remote Sens., 25(1), 1-8. https:// doi.org/10.1080/01431160310001642 395

Ballestero, D., Márquez, A., Salazar, P. \& Murillo, G. (2012). Condiciones Oceanográficas en el Golfo de Papagayo. Informe Técnico. Universidad Nacional, Laboratorio de Oceanografía. Heredia: Costa Rica.

Brenes, C. L., Lavin, M. F. \& Mascarenhas, A. (2008). Geostrophic circulation between the Costa Rica Dome and Central America. Deep Sea Res., I 55, 608-629. https://doi.org/10.1016/j. dsr.2008.02.005

Caron, D. A. (2000). Symbiosis and mixotrophy among pelagic microorganisms. In D. A. Kirchman (Ed.), Microbial Ecology of the Oceans (pp. 495-523). New York, EE. UU.: John Wiley and Sons.

Chelton, D. B., Freilich, M. H. \& Esbensen, S. K. (2000a). Satellite Observations of the Wind Jets off the Pacific coast of Central America. Part I: Case studies and statistical characteristics. Mon. Wea. Rev., 128, 19932018. https://doi.org/10.1175/1520$0493(2000) 128<1993$ : SOOTWJ $>2.0 . \mathrm{CO} ; 2$

Chelton, D. B., Freilich, M. H. \& Esbensen, S. K. (2000b). Satellite Observations of the Wind Jets off the Pacific Coast of Central America. Part II: Regional Relationships and Dynamical Considerations. Mon. Wea. Rev., 128, 2019-
2043. https://doi.org/10.1175/1520$0493(2000) 128<1993$ : SOOTWJ $>2.0 . \mathrm{CO} ; 2$

De la Lanza, G., Hernández, S. \& Carvajal, J. L. (2000). Organismos indicadores de la calidad del agua y de la contaminación (bioindicadores). México D. F, México.: Plaza \& Valdés, S. A.

Estrada, M. (1982). Ciclo anual del fitoplancton en la zona costera frente a Punta Endata (Golfo de Vizcaya). Inv. Pesq., 46(3), 469-491.

Fiedler, P. (2002). The annual cycle and biological effects of the Costa Rica Dome. Deep-Sea Res., I (49): 231-338. https://doi.org/10.1016/S0967-0637 (01)00057-7

Fiedler, P. C. \& Talley, L. D. (2006). Hydrography of the eastern Pacific: A review. Progr. Oceanogr., 69, 143-180. https:// doi.org/10.1016/j.pocean.2006.03.008

Friligos, N. \& Koussouris, T. (1984). Preliminary observations on sewage nutrient enrichment and phytoplankton ecology in the Thermaikos Gulf, Thessalonik, Greece. Vie et Milieu, 31(1), 35-39.

Friligos, N. (1989). Hydrographic and plankton variability in the Saronikos Gulf. Toxicol. Envirom. Chem., 24, 155-170. https://doi. org/10.1080/02772248909357486

Frontier, S. (1981). Recuentos y análisis de los datos. Tratamiento de los datos. En D. Boltovskoy (Ed.), Atlas del zooplancton del Atlántico Sudoccidental y método de trabajo con zooplancton marino (pp. 169-188). Mar del Plata, Argentina: INIDEP.

Gómez, F. (2013). Reinstatement of the dinoflagellate genus Tripos to replace Neoceratium, marine species of Ceratium (Dinophyceae, Alveolata). CICIMAR. Oceánides, 28(1), 1-22.

GRAPHER 8. (2009). Grapher ${ }^{\circledR} 2 \mathrm{D}$ and 3D Graphing Software for Scientists and 
Engineers, version 8www.GoldenSoftware.com. Colorado, EE. UU.: Golden Software, Inc.

Guiry, M. D. \& Guiry, G. M. (2017). Algae Base. World-wide electronic publication, National University of Ireland, Galway. Recuperado en julio 07, 2017, disponible en http://www.algaebase.org.

Herrera-Silveira, J., Martín, M. \& Díaz-Arce, V. (1999). Variaciones del fitoplancton en cuatro lagunas costeras del Estado de Yucatán, México. Rev. Biol. Trop., 47(1), 47-56.

IMN. (2008). Instituto Meteorológico Nacional. Clima en Costa Rica: Pacífico Norte. Recuperado en setiembre 09, 2017, disponible en https://www.imn.ac.cr/ documents/10179/31165/PacificoNorte.pdf/4a0e8960-8c51-4390-8a8d$73 \mathrm{~d} 9 \mathrm{~d} 825 \mathrm{~d} 59 \mathrm{~b}$.

Józwiak, T., Mazur-Marzec, H. \& Plinski, M. (2008). Cyanobacterial blooms in the Gulf of Gdan'sk (southern Baltic): The main effect of eutrophication. Oceanol. Hydrobiol. Stud., 37, 115-121. https:// doi.org/10.2478/v10009-008-0015-Z

Koblentz-Mishke, O. J. \& Vedernikov, V. I. (1979). Producción Primaria. En M. E. Vinogradov (Ed.), Oceanología, Biología del Océano (pp. 183-309). Nauka, Moscú: Producciones Biológicas del Océano.

Licea-Durán, S., Moreno-Ruiz, J. L., Santoyo, H. \& Figueroa, G. (1995). Dinoflagelados del Golfo de California. México: $\mathrm{UABCS}$.

Loza, S., Lugioyo, M., Martínez, M., Miravet, M. E., Montalvo, J. \& Sánchez, M. (2007). Evaluación de la calidad de las aguas del Golfo de Batabanó a partir de indicadores biológicos y químicos. Rev. Invest. Mar., 28(2), 111-120.

May-Collado, L. \& Morales, R. A. (2005). Presencia y patrones de comportamiento del delfín manchado costero, Stene- lla attenuata (Cetacea: Delphinidae) en el Golfo de Papagayo, Costa Rica. Rev. Biol. Trop., 53(1-2), 265-276.

Miravet, M. E., Lugioyo, M., Loza, S., Enríquez, D., Delgado, Y., Carmenate, M. \& Pérez, D. M. (2009). Procedimientos para el monitoreo de la calidad ambiental en la zona marino costera a partir de microorganismos. La Habana, Cuba: Centenario.

Morales, R. A., Víquez, R. R., Vargas, K. \& Vargas, M. (2001). Marea Roja producida por Lingulodinium polyedrum (Peridiniales, Dinophyceae) en Bahía Culebra, Golfo de Papagayo, Costa Rica. Rev. Biol. Trop., 49(2), 19-23.

Moreira, A., Seisdedo, M., Leal, S., Comas, A., Delgado, G., Regadera, R., Alonso, C., Muñoz, A. \& Abatte, M. (2007). Composición y abundancia del fitoplancton de la Bahía Cienfuegos, Cuba. Rev. Invest. Mar., 28(2), 97-109.

Moreno-Ruíz, J. L., Licea-Durán, S. \& Santoyo, H. (1996). Diatomeas del Golfo de California. Universidad Autónoma de Baja California Sur, México: SEP-FOMES-PROMARCO.

Paz, B., Daranas, A. H., Norte, M., Riobó, P., Franco, J. M. \& Fernández, J. J. (2008). Yessotoxins, a Group of Marine Polyether Toxins: an Overview. Mar. Drugs., 6, 73-102. https://doi.org/10.3390/md 20080005

Potapova, N. A. (1993). Destruction of organic matter in different size fractions of plankton in lower Dniester and Deniester liman. Gidrobiol. Zh. Hydrobiol. J., 29(2), 12-22.

Salas, R. A. (2010). Polo Turístico Golfo de Papagayo, Guanacaste, Costa Rica. Análisis del Polo Turístico Golfo de Papagayo como un modelo único gubernamental de concesión turística. En Center for Responsible Travel (Eds.), The Impact of Tourism Related Develo- 
pment along Costa Rica's Pacific Coast (pp. 36-46). Washington, EE. UU.: Center for Responsible Travel.

Sánchez-Noguera, C. (2012). Entre historias y culebras: más que una bahía (Bahía Culebra, Guanacaste, Costa Rica). Rev. Biol. Trop., 60(2), 1-17.

Sant'Anna, C., Azevedo, M. T., Agujaro, L., Carvalho, M. C. \& Souza, R. C. (2005). Manual ilustrado para identificación y conteo de cianobacterias planctónicas de aguas continentales brasileiras. Sao Paulo, Brasil: Interciencia.

Sar, E. A., Ferrario, M. E. \& Reguera, B. (2002). Floraciones algales nocivas en el Cono Sur Americano. Madrid, España: Instituto Español de Oceanografía.

Simon, N., Cras, A. L., Foulon, E. \& Lemee, R. (2009). Diversity and evolution of marine phytoplankton. C. R. Biol., 332, 159-170. https://doi.org/10.1016/j. crvi.2008.09.009

Smayda, T. \& Shimizu, Y. (1993, noviembre). Toxic Phytoplankton Blooms in the Sea. Proceedings of the Fifth International Conference on Toxic Marine Phytoplankton, Newport, EE. UU.

SURFER 14 (2017). Surfer ® Contouring and 3D Surface Mapping for Scientists and Engineers, version 14 www.Golden-
Software.com. Colorado, EE.UU: Golden Software, Inc.

Tomas, C. R. (1997). Identifying Marine Diatoms and Dinoflagellates. New York, EE. UU.: Academic Press.

Vargas, C. (2013). Contaminación fecal amenaza el turismo en Playas del Coco. La Nación. Recuperado en julio 13, 2017, disponible en http://www. nacion.com/nacional/Contaminacion-amenazael-turismo-Playas-coco_0_1353464668.html

Vilicic, D., Krsinic, F., Caric, M., Jasprica, N., Bobanovic, S. \& Mikus, J. (1995). Plankton and hydrography in a moderately eutrophicated eastern Adriatic bay (Gruz Bay). Hydrobiologia, 304, 9-22. https://doi.org/10.1007/BF00006876

Wo Ching, S. E. \& Moreno, C. (2001). Evaluación nacional sobre fuentes de contaminación y actividades humanas originadas en tierra que afectan ambientes marinos, costeros y dulce acuícola asociados en Costa Rica. Informe Centro de Derecho Ambiental y Recursos Naturales CEDARENA/GPA, San José, Costa Rica. Sin editorial. 